\title{
Auditoria em enfermagem: revisão sistemática da literatura
}

\author{
Nursing audit: systematic review of the literature \\ Auditoría en enfermería: revisión sistemática de la literatura
}

\section{Teresa Cristina Lyporage Dias', José Luís Guedes dos Santos", Onélia da Costa Pedro Cordenuzzi"I, Adelina Giacomelli Prochnow ${ }^{\text {IV }}$}

\author{
' Hospital da Guarnição. Santa Maria-RS, Brasil. \\ "Universidade Federal do Rio Grande do Sul, Programa de Pós-Graduação em Enfermagem. (Mestrando) Porto Alegre-RS, Brasil. \\ III Universidade Federal de Santa Maria, Programa de Pós-Graduação em Enfermagem. (Mestranda) Santa Maria-RS, Brasil. \\ IV Universidade Federal de Santa Maria, Departamento de Enfermagem, \\ Coordenação do Programa de Pós-Graduação em Enfermagem. Santa Maria-RS, Brasil.
}

Submissão: 20-12-2009 Aprovação: 29-05-2011

\section{RESUMO}

Este estudo teve como objetivo analisar a produção científica acerca da auditoria em enfermagem publicada em artigos nacionais entre 1998 e 2008. Realizou-se uma revisão sistemática da literatura, com os artigos sendo pesquisados nas bases de dados LILACS e SciELO, a partir dos descritores auditoria, auditoria de enfermagem, qualidade da assistência à saúde e supervisão de enfermagem. A amostra constituiu-se de dez trabalhos. As publicações analisadas descrevem as principais distorções e não conformidades presentes nas anotações de enfermagem, relacionando-as, principalmente, com a qualidade da assistência de enfermagem e com os prejuízos financeiros às instituições. As publicações também destacam a função administrativa do enfermeiro, sobretudo a função de liderança junto à equipe de enfermagem; e a auditoria de enfermagem, como um processo educativo.

Descritores: Enfermagem; Auditoria de enfermagem; Qualidade da assistência à saúde; Supervisão de enfermagem.

\section{ABSTRACT}

This study aimed to analyze the scientific production about audit in nursing, published in national papers, between 1998 and 2008. A systematic review of the literature was conducted, with articles being searched in the databases LILACS and SciELO, from the key words: audit, nursing, quality health care and nursing supervision. The sample consisted of ten works. The analyzed publications describe the main distortions and non-conformities found in nursing records, relating them mainly with the quality of nursing care and with the financial damage to the institutions. The publications also include the administrative role of the nurse, especially her/his leadership role in the team of nursing; and nursing audit as an educational process

Key words: Nursing Audit; Quality of Health Care; Nursing, Supervisory.

\section{RESUMEN}

Este estudio tuvo el objetivo de analizar la producción científica sobre la auditoría en enfermería publicada en artículos nacionales entre 1998 y 2008. Esta es una revisión sistemática de la literatura. Los artículos fueron buscados en las bases de datos LILACS y SciELO a partir de los descriptores auditoría, la auditoría de enfermería, atención de salud de calidad y supervisión de enfermería, constituyéndose una muestra de 10 trabajos. Las publicaciones analizadas describen las principales distorsiones e inconformidades presentes en las notas de enfermería relacionándolas, principalmente, con la calidad de los cuidados de enfermería y los prejuicios financieros. También destacan la función administrativa de los enfermeros, en especial su papel como líder del equipo de enfermería y la auditoría en enfermería como un proceso educativo.

Palabras clave: Auditoria de Enfermería; Calidad de la Atención de Salud; Supervisión de Enfermería. 


\section{INTRODUÇÃO}

A palavra auditoria origina-se do latim audire que significa ouvir. No entanto, o termo pode ser melhor explicado pela palavra da língua inglesa audit, o qual tem o sentido de examinar, corrigir e certificar. Sendo assim, a auditoria consiste na avaliação sistemática e formal de uma atividade para determinar se ela está sendo realizada de acordo com os seus objetivos. Trata-se de um ramo da contabilidade que tem sido utilizada por várias profissões, inclusive pela enfermagem, devido à globalização e dada a necessidade das empresas de somarem o trabalho do auditor de enfermagem ao auditor médico, considerando sua função generalista $^{(1,2)}$.

Nas organizações de saúde, a auditoria configura-se como uma importante ferramenta na transformação dos processos de trabalho que vêm ocorrendo em hospitais e operadoras de planos de saúde, os quais estão buscando se reestruturar para manterem a qualidade do cuidado prestado e ao mesmo tempo garantirem uma posição competitiva no mercado de trabalho. Nesse contexto, a auditoria em enfermagem pode ser definida como "a avaliação sistemática da qualidade da assistência de enfermagem, verificada através das anotações de enfermagem no prontuário do paciente e/ou das próprias condições deste ${ }^{\prime \prime(1)}$.

A auditoria de enfermagem vem atender às necessidades das instituições de saúde no controle dos fatores geradores de processos de alto custo. No âmbito hospitalar, por exemplo, a enfermagem é usuária da maior parte dos materiais de consumo, devendo dispor atenção aos custos envolvidos no processo de cuidar, no intuito de garantir a provisão e adequação dos materiais de uso e, principalmente, da qualidade da assistência de enfermagem ${ }^{(3)}$. As principais finalidades da auditoria de enfermagem são identificar áreas deficientes dos serviços de enfermagem, fornecendo dados concretos para que decisões sejam tomadas em relação ao remanejamento e aumento de pessoal, possibilitando, consequentemente, melhoria do cuidado de enfermagem ${ }^{(1)}$.

Com o foco na assistência de qualidade e no aumento da competitividade entre organizações que prestam serviços de saúde, cada vez mais surgem oportunidades para o profissional enfermeiro atuar na área de auditoria de contas hospitalares. Desse modo, a auditoria configura-se como uma ferramenta gerencial utilizada pelos profissionais da saúde, em especial os enfermeiros, com a finalidade de avaliar a qualidade da assistência de enfermagem e os custos decorrentes da prestação dessa atividade ${ }^{(4)}$.

Frente ao panorama exposto, este estudo teve como objetivo analisar a produção científica acerca da auditoria em enfermagem publicada em artigos nacionais entre 1998 e 2008. Espera-se, com este trabalho, contribuir com as investigações que vêm sendo realizadas acerca da temática, pois uma das tendências para o gerenciamento dos serviços de enfermagem é a inclusão de conhecimentos sobre custos, como uma importante ferramenta a ser utilizada nos processos de tomada de decisão dos enfermeiros ${ }^{(5)}$.

\section{ASPECTOS METODOLÓGICOS}

Trata-se de um estudo de revisão sistemática da literatura, que é adequado para buscar consenso sobre alguma temática específica e sintetizar o conhecimento de uma dada área por meio da formulação de uma pergunta, identificação, seleção e avaliação crítica de estudos científicos contidos em bases de dados eletrônicas. A partir desse processo, ela permite, além de aprofundar o conhecimento sobre a temática investigada, apontar lacunas que precisam ser preenchidas por meio da realização de novas investigações ${ }^{(6,7)}$.

A pergunta de pesquisa foi: qual é o conhecimento científico já produzido, no Brasil, sobre a auditoria de enfermagem? A busca de artigos foi realizada nas bases eletrônicas Literatura Latino-Americana em Ciências da Saúde (LILACS) e Scientific Electronic Library Online (SciELO), por meio das palavras-chaves selecionadas segundo a classificação dos Descritores em Ciências da Saúde (DeCS): auditoria, auditoria de enfermagem, qualidade da assistência à saúde e supervisão de enfermagem.

Para seleção dos artigos realizou-se, primeiramente, a leitura dos resumos das publicações selecionadas com o objetivo de refinar a amostra por meio de critérios de inclusão e exclusão. Foram incluídos artigos originais publicados entre 1998 e 2008 e oriundos de estudos desenvolvidos no Brasil. Os critérios de exclusão foram: artigos de revisão ou reflexão e ausência de resumo nas plataformas de busca on-line. Por meio desse processo, a amostra final foi constituída por dez artigos.

A avaliação crítica dos artigos consistiu na leitura do estudo na íntegra e, em seguida, na elaboração de quadros sinópticos com os dados coletados com informações de cada pesquisa, a saber: autores/data/periódico, objetivo da pesquisa, tipo de estudo, aspectos metodológicos, principais resultados e conclusões ${ }^{(6)}$. De forma auxiliar, fez-uso da técnica de análise temática de conteúdo por meio da leitura e releitura dos resultados dos estudos, procurando identificar aspectos relevantes que se repetiam ou se destacavam ${ }^{(8)}$. Para descrever os achados referentes aos dados analisados, também foi realizada análise estatística descritiva por meio de cálculos de frequência simples.

\section{RESULTADOS}

Nesta pesquisa bibliográfica foram analisados dez artigos científicos que atenderam aos critérios de inclusão estabelecidos previamente. Para facilitar a análise e apresentação dos resultados, elaborou-se o Quadro 1 com dados sobre o ano, tipo de produção, objetivos e resultados e ou conclusões de cada estudo.

Oito dos artigos analisados foram publicados entre os anos de 2002 e 2008. Nesse sentido, cabe destacar que as atividades do enfermeiro auditor foram regulamentadas pelo Conselho Federal de Enfermagem (Cofen) em 2001, por meio da Resolução Cofen $N^{\circ}$ 266/2001, o que pode ter estimulado o aumento das investigações relacionadas à auditoria de enfermagem nos últimos anos ${ }^{(19)}$. Outrossim, deve-se considerar que 


\begin{tabular}{|c|c|c|}
\hline Código/Ano/Tipo & Objetivo(s) & Resultados/Conclusões \\
\hline $\begin{array}{l}\text { Artigo } 1^{(9)} \\
\quad 1998 \\
\text { Documental }\end{array}$ & $\begin{array}{l}\text { Analisar as distorções oriundas das } \\
\text { auditorias em Unidades Ambulatoriais } \\
\text { e Hospitalares de Saúde do Estado do } \\
\text { Acre, no ano de 1994/95, com ênfase } \\
\text { nas anotações de enfermagem. }\end{array}$ & $\begin{array}{l}\text { Encontraram-se } 491 \text { distorções, sendo } 20,2 \% \text { em Enfermagem: } 15,1 \% \\
\text { ocorreram na administração de medicamentos e } 34,3 \% \text { em anotações. } \\
\text { As regras de anotações não vêem sendo seguidas pelos profissionais de } \\
\text { enfermagem, colocando em risco paciente, profissional e a própria instituição. }\end{array}$ \\
\hline $\begin{array}{l}\text { Artigo } 2^{(10)} \\
2001 \\
\text { Documental }\end{array}$ & $\begin{array}{l}\text { Analisar as anotações de enfermagem, } \\
\text { realizadas pela equipe de enfermagem } \\
\text { de uma unidade hospitalar, segundo } \\
\text { aparência e conteúdo. }\end{array}$ & $\begin{array}{l}\text { O auxiliar de enfermagem realiza o maior número de anotações extensas e } \\
\text { pouco específicas. Já nos registros realizados pelo enfermeiro observou-se } \\
\text { maior número de anotações legíveis e objetivas. As anotações de enfermagem } \\
\text { precisam ser revistas e reordenadas, especialmente ao registrarem } \\
\text { informações importantes relacionadas à condição do paciente, com vistas a } \\
\text { uma melhor documentação das ações e intervenções realizadas pela equipe } \\
\text { de enfermagem. Cabe aos enfermeiros liderar a concretização dessa melhora. }\end{array}$ \\
\hline $\begin{array}{l}\text { Artigo } 3^{(11)} \\
\quad 2002 \\
\text { Documental e } \\
\text { descritivo }\end{array}$ & $\begin{array}{l}\text { Registrar anomalias na assistência de } \\
\text { enfermagem; identificar e classificar } \\
\text { suas causas e conseqüências; oferecer } \\
\text { subsídios para o planejamento e } \\
\text { implementação de atividades de } \\
\text { orientação e treinamento; fornecer } \\
\text { indicadores para a gerência de } \\
\text { enfermagem. }\end{array}$ & $\begin{array}{l}\text { Os dados demonstraram que o maior índice de anomalias relaciona-se ao } \\
\text { item registro tendo como principal causa as falhas de método e processo. } \\
\text { Os maiores índices de anomalias foram: registro das condições do paciente } \\
(41 \%) \text {, necessidades terapêuticas ( } 20 \%) \text { e organização da unidade e conforto } \\
\text { do paciente }(10 \%) \text {. Esses itens estão relacionados à falha humana e ausência } \\
\text { de métodos e processos de trabalho como também à falta de treinamento. }\end{array}$ \\
\hline $\begin{array}{l}\text { Artigo } 4^{(12)} \\
\quad 2002 \\
\text { Estudo de caso } \\
\text { múltiplo }\end{array}$ & $\begin{array}{l}\text { Estudar o papel da auditoria } \\
\text { de enfermagem para a redução } \\
\text { dos desperdícios em materiais e } \\
\text { medicamentos. }\end{array}$ & $\begin{array}{l}\text { Foram verificados os registros incorretos da equipe de enfermagem com } \\
\text { relação aos medicamentos e materiais. Os setores de auditoria de enfermagem } \\
\text { não realizam um trabalho proativo para a redução de desperdícios } \\
\text { hospitalares em materiais e medicamentos, sendo necessária a revisão de suas } \\
\text { rotinas, bem como a implantação de um treinamento e a conscientização da } \\
\text { equipe de enfermagem, a fim de que todos sejam informados dos recursos } \\
\text { econômico-financeiros do hospital. }\end{array}$ \\
\hline $\begin{array}{l}\text { Artigo } 5^{(13)} \\
2004 \\
\text { Exploratório e } \\
\text { descritivo }\end{array}$ & $\begin{array}{l}\text { Investigar os fatores intervenientes nas } \\
\text { glosas ocorridas em um hospital de } \\
\text { ensino. }\end{array}$ & $\begin{array}{l}\text { Foram encontrados } 17.324 \text { itens glosados nos } 85 \text { recursos de glosas } \\
\text { analisados, os quais representaram um valor de } \mathrm{R} \$ 31.856,52 \text {. Os recursos } \\
\text { de glosas para materiais representaram } 55 \% \text { do valor total. Cerca de } 99,6 \% \\
\text { dos recursos de glosas para materiais foram embasados nas "anotações e/ou } \\
\text { checagem de enfermagem". Destaca a função administrativa do enfermeiro e } \\
\text { cooperem com o resultado econômico nas instituições de saúde. }\end{array}$ \\
\hline $\begin{array}{l}\text { Artigo } 6^{(14)} \\
2005 \\
\text { Exploratório e } \\
\text { descritivo }\end{array}$ & $\begin{array}{l}\text { Relatar a importância da atuação da } \\
\text { auditoria de enfermagem e o uso de } \\
\text { indicadores assistenciais como um dos } \\
\text { métodos de avaliação da qualidade de } \\
\text { assistência de enfermagem prestada } \\
\text { aos clientes. }\end{array}$ & $\begin{array}{l}\text { Não-conformidades: prescrição de enfermagem ( } 26,3 \%) \text {, falta de checagem na } \\
\text { prescrição médica }(20,43 \%) \text {, falta de checagem na prescrição da enfermeira } \\
(18,77 \%) \text { e falta de registro na realização do curativo }(10,6 \%) \text {. A participação } \\
\text { das lideranças de enfermagem no planejamento e à adoção de medidas } \\
\text { corretivas e preventivas é importante para a gestão do serviço de enfermagem } \\
\text { e contribuir para solidez da excelência na gestão hospitalar. }\end{array}$ \\
\hline $\begin{array}{l}\text { Artigo } 7^{(15)} \\
\quad 2005 \\
\text { Quantitativo e } \\
\text { descritivo }\end{array}$ & $\begin{array}{l}\text { Identificar a opinião de enfermeiros } \\
\text { sobre auditoria }\end{array}$ & $\begin{array}{l}\text { Auditoria significa interface entre o quântico-ecnonômico-financeiro } \\
\text { e legislações afim. O papel do enfermeiro auditor é gerenciamento da } \\
\text { assistência. É uma área em expansão, especialmente para profissionais } \\
\text { experientes. O âmbito hospitalar destaca-se como principal campo de } \\
\text { atuação. }\end{array}$ \\
\hline $\begin{array}{l}\text { Artigo } 8^{(16)} \\
\quad 2007 \\
\text { Quantitativo, } \\
\text { descritivo e } \\
\text { exploratório }\end{array}$ & $\begin{array}{l}\text { Identificar a qualidade dos registros de } \\
\text { enfermagem em contas hospitalares }\end{array}$ & $\begin{array}{l}\text { Principais problemas encontrados: anotações realizadas por turno e não por } \\
\text { horário; rasuras nas escritas; espaços em branco ao longo do impresso; falta } \\
\text { de carimbo e de assinatura. Verificou-se prontuários em que a checagem } \\
\text { de prescrições não ocorre ou é realizada de forma incorreta; anotação } \\
\text { incompleta de sinais vitais. }\end{array}$ \\
\hline $\begin{array}{l}\text { Artigo } 9^{(17)} \\
2008 \\
\text { Técnica Delphi }\end{array}$ & $\begin{array}{l}\text { Identificar e analisar opiniões } \\
\text { de especialistas em auditoria e } \\
\text { sistematizar tendências de concepção, } \\
\text { método e finalidade da auditoria em } \\
\text { enfermagem na atualidade e para os } \\
\text { próximos cinco anos. }\end{array}$ & $\begin{array}{l}\text { A concepção atual da auditoria está enfocada na visão contábil e financeira, } \\
\text { tendo em vista a sustentação econômica do hospital e como ato de } \\
\text { controladoria visando identificar pagamentos indevidos referentes à conta } \\
\text { hospitalar; no futuro, essa concepção será associada à avaliação da qualidade } \\
\text { da assistência, com envolvimento em outras áreas que nela interferem. }\end{array}$ \\
\hline $\begin{array}{l}\text { Artigo } 10^{(18)} 2008 \\
\text { Estudo inter- } \\
\text { relacional } \\
\text { retrospectivo }\end{array}$ & $\begin{array}{l}\text { Analisar a qualidade das anotações } \\
\text { de enfermagem acerca dos cuidados } \\
\text { dispensados ao paciente cirúrgico. }\end{array}$ & $\begin{array}{l}\text { Apenas os registros referentes às prescrições de enfermagem de pós- } \\
\text { operatório, observação de sinais e sintomas e anotações de pós-operatório, } \\
\text { puderam ser consideradas completas. As anotações referentes ao aspecto e } \\
\text { evolução das lesões cutâneas e as anotações de alta estavam incompletas em } \\
73,9 \text { por cento e } 97 \text { por cento dos registros analisados. Pontua a necessidade } \\
\text { de educação, monitoramento e avaliação contínua dos registros de } \\
\text { enfermagem com vistas à melhoria dos mesmos e da assistência prestada. }\end{array}$ \\
\hline
\end{tabular}

Quadro 1 - Identificação dos artigos 
as transformações ocorridas no mundo do trabalho em saúde e enfermagem, como o déficit orçamentário nas instituições públicas, e o incremento das iniciativas privadas de atenção à saúde, tornam a relação entre a auditoria, os custos e a qualidade da assistência uma problemática cada vez mais atual.

Com relação ao periódico de publicação, destacou-se a Revista Brasileira de Enfermagem com a publicação de três artigos referentes à temática. Salienta-se que outros três artigos da amostra constituída são oriundos de revistas multidisciplinares, que abrangem publicações das diversas áreas da saúde.

No que tange às questões de autoria, destacaram-se artigos publicados por três ou mais autores, entre os quais a grande maioria enfermeiros (23) e um administrador hospitalar. Esses autores, quanto à titulação acadêmica, eram, majoritariamente, Doutores, Mestres, Mestrandos e Especialistas.

Entre os artigos selecionados, os principais objetivos apresentados foram identificar e analisar a qualidade das anotações de enfermagem, destacando as principais distorções que ocorrem, apontando suas possíveis causas e as repercussões que elas geram na avaliação da assistência de enfermagem e na gestão de custos pelas instituições de saúde. Por meio desses objetivos, os autores também destacam a pretensão de fornecer subsídios para o planejamento e execução de atividades gerenciais, como orientação e capacitação da equipe de enfermagem para execução dos registros de enfermagem. Apenas um artigo objetivou conhecer a opinião dos enfermeiros acerca da auditoria em enfermagem.

Quanto aos aspectos metodológicos, observou-se destaque da utilização da abordagem quantitativa. Em relação ao tipo de estudo eles são majoritariamente exploratório-descritivos (6), apenas descritivos (2), um (1) estudo de casos múltiplos e em uma pesquisa os autores utilizam a técnica Delphi.

A pesquisa quantitativa relaciona-se à dimensão material da realidade e tem como objetivo traduzir em números opiniões e informações para classificá-las e analisá-las, por meio da utilização de recursos e de técnicas estatísticas. Seus resultados auxiliam o planejamento de ações coletivas e são passíveis de maior generalização, principalmente quando a amostra representa com fidelidade a população estudada ${ }^{(20,21)}$.

Em relação aos tipos de estudos, cabe explicitar que os exploratórios permitem ao pesquisador aumentar sua experiência em torno de um determinado problema, a partir da busca de maior conhecimento a seu respeito, e os de caráter descritivo tem como objetivo descrever com maior exatidão fatos e fenômenos de uma determinada realidade ${ }^{(21)}$. Já o estudo de caso, é uma modalidade de investigação empírica mais abrangente, que se estrutura por meio da lógica do planejamento, da coleta e da análise dos dados visando obter maior conhecimento de um fenômeno estudado. Pode ter foco em uma unidade - um indivíduo (caso único e singular, como o caso clínico) ou múltiplo, nos quais vários estudos são conduzidos simultaneamente, como por exemplo: vários indivíduos ou várias organizações ${ }^{(22)}$. A técnica Delphi é uma ferramenta de pesquisa que se baseia na consulta a um grupo de especialistas sobre eventos futuros por meio de questionários, os quais são repassados repetidas vezes até a obtenção de convergências entre as respostas ${ }^{(23)}$.
Os hospitais foram os principais cenários investigados, com destaque para instituições privadas (6) e hospitais escola (2). A maior produção de estudos em organizações de saúde de caráter privado pode estar relacionada às metas que elas estabelecem com o objetivo de evitar desperdícios, a partir da redução de custos e cobrança de todos os procedimentos e equipamentos utilizados nas atividades assistenciais desenvolvidas.

Entretanto, um estudo bibliográfico ${ }^{(24)}$ que buscou descrever os conceitos de auditoria de enfermagem utilizados e analisar os contextos em que eles estão inseridos entre 1988 - 2003, nos portais de busca BDENF, Biblioteca Virtual da Saúde e Revista Nursing Brasileira, não encontrou estudos voltados para as instituições particulares de saúde contatando a escassez de estudos no âmbito privado de atenção à saúde. Essa divergência pode estar relacionada às bases de dados consultadas ou mesmo ao marco temporal estabelecido, o que nos permite inferir um avanço nas investigações relacionadas à auditoria em enfermagem.

A maioria dos estudos utilizou bases documentais (8) para obtenção dos dados, principalmente consulta retrospectiva aos prontuários e/ou registros de anotações da equipe de enfermagem. Para a coleta de dados, os pesquisadores utilizaram instrumentos próprios, como formulários e planilhas elaborados a partir das informações que desejavam pesquisar. A ausência de padrões e falta de clareza na descrição do método empregado nas pesquisas relacionadas à auditoria em enfermagem também foi destacada em um dos estudos ${ }^{(17)}$.

Dessa forma, a não uniformidade entre os aspectos elencados para estudo entre as publicações, dificulta a comparação dos resultados apresentados. Entretanto, mister é salientar que os delineamentos metodológicos dos estudos são coerentes com os objetivos elencados e os resultados descritos, o que permite a análise da produção do conhecimento acerca da temática.

Os resultados de oito dos estudos analisados descrevem as principais distorções e não conformidades (Quadro 2) presentes nas anotações de enfermagem, relacionando-as com a qualidade da assistência de enfermagem ${ }^{(10,11,12,14)}$; as implicações éticas e legais aos profissionais e à instituição ${ }^{(9,12)}$; os prejuízos financeiros à instituição ${ }^{(9,13)}$; a visibilidade do trabalho da enfermagem ${ }^{(10)}$; e a necessidade de educação, monitoramento e avaliação contínua dos registros de enfermagem com vistas à melhoria dos mesmos e da assistência prestada ${ }^{(18)}$.

A auditoria hospitalar permite uma avaliação dos aspectos qualitativos e quantitativos relacionados à assistência. Também envolve a observação de aspectos organizacionais, operacionais e financeiros, mas sempre com o mesmo foco: a qualidade dos cuidados prestados ${ }^{(25)}$. As principais distorções e não conformidades presentes nas anotações de enfermagem sintetizadas no quadro acima podem comprometer a qualidade dos atos cuidadores de enfermagem e mesmo comprometer a avaliação da evolução terapêutica dos pacientes, tais como o não aprazamento das medicações administradas e dos curativos realizados.

A crença equivocada de que o registro não é prioridade na execução do trabalho da enfermagem é muito mais uma 


\begin{tabular}{|c|c|}
\hline Quesito & Código \\
\hline $\begin{array}{l}\text { Solicitação e aprazamento da administração de } \\
\text { medicamentos }\end{array}$ & $\begin{array}{l}\text { Artigo } 1^{(9)} \\
\text { Artigo } 4^{(12)} \\
\text { Artigo } 5^{(13)}\end{array}$ \\
\hline Letra pouco legível e rasuras & $\begin{array}{l}\text { Artigo } 1^{(9)} \\
\text { Artigo } 2^{(10)} \\
\text { Artigo } 8^{(16)}\end{array}$ \\
\hline $\begin{array}{l}\text { Falta de checagem na prescrição médica e de } \\
\text { enfermagem }\end{array}$ & $\begin{array}{l}\text { Artigo } 6^{(14)} \\
\text { Artigo } 8^{(16)}\end{array}$ \\
\hline Anotações extensas e pouco específicas & $\begin{array}{l}\text { Artigo } 1^{(9)} \\
\text { Artigo } 2^{(10)}\end{array}$ \\
\hline $\begin{array}{l}\text { Condições de pacientes, necessidades } \\
\text { terapêuticas, organização da unidade e conforto } \\
\text { do paciente }\end{array}$ & $\begin{array}{l}\text { Artigo } 1^{(9)} \\
\text { Artigo } 3^{(11)}\end{array}$ \\
\hline $\begin{array}{l}\text { Falta de registro/registros incompletos da } \\
\text { realização de curativos }\end{array}$ & $\begin{array}{l}\text { Artigo } 6^{(14)} \\
\text { Artigo } 10^{(18}\end{array}$ \\
\hline Anotações por turno e não por horário & Artigo $8^{(16)}$ \\
\hline
\end{tabular}

Quadro 2 - Principais distorções e não conformidades presentes nas anotações de enfermagem

questão cultural do que técnica. Porém, quando se discute a importância das anotações, deve-se considerar a escassez de pessoal de enfermagem que é a realidade de muitos hospitais do Brasil e do mundo e o ritmo de trabalho da enfermagem. Esses fatores dificultam o registro das informações e representam uma questão chave para melhorar a qualidade das anotações de enfermagem ${ }^{(26)}$.

Nesse sentido, cabe salientar que o prontuário é um documento legal em que devem constar as informações pertinentes a sua internação e aos procedimentos que são realizados pelos profissionais de saúde envolvidos no atendimento ${ }^{(27)}$.

Um estudo identificou a opinião dos enfermeiros sobre auditoria, com aplicação de um questionário, evidenciando que o papel do enfermeiro é o gerenciamento da assistência, por meio da mediação entre as interfaces quântico-econômico-financeira e legislações relacionadas ${ }^{(15)}$. O âmbito hospitalar foi apontado como o principal campo de atuação e a área de auditoria como em expansão, principalmente, para profissionais experientes.

A finalidade da auditoria em enfermagem, na atualidade, está relacionada principalmente à comprovação de pagamento de contas hospitalares, revendo glosas por meio da elaboração de relatórios técnicos e realizando negociações entre representantes do hospital e de convênios. No futuro, associado à primeira finalidade, a intenção será apontar inadequações da assistência de enfermagem, reformulando suas práticas, indicando processos de educação em serviço e delineando ações corretivas ${ }^{(17)}$.

Três estudos destacam a atuação gerencial do enfermeiro na auditoria de enfermagem, sobretudo a sua função de liderança junto à equipe de enfermagem ${ }^{(12-14)}$.
É responsabilidade do enfermeiro o planejamento e organização da unidade e do trabalho da equipe de enfermagem, o que inclui maior atenção ao registro da história do paciente no seu prontuário, visando à diminuição dos desperdícios e maior qualidade do atendimento ${ }^{(12)}$. De forma semelhante, outro estudo salienta que o enfermeiro no exercício da sua função administrativa deve cooperar com os resultados econômicos da instituição ${ }^{(13)}$, a participação das lideranças de enfermagem é importante na adoção de medidas corretivas e preventivas no que tange aos registros de enfermagem e podem conduzir à excelência na gestão hospitalar ${ }^{(14)}$.

Embora a importância das anotações de enfermagem seja um assunto presente e bastante enfatizado nos cursos de formação de técnicos de enfermagem e enfermeiros, pesquisa identificou que são comuns erros e inconformidades na sua elaboração. Diante disso, cogita-se que a falha pode estar na Direção de Enfermagem da instituição ou nos enfermeiros diretamente envolvidos com os serviços ou no rodízio em turnos de trabalhos, que pode contribuir para a não continuidade de anotações de enfermagem e impedir que os enfermeiros acompanhem seus funcionários mais de perto(9).

Outro aspecto em comum entre os artigos analisados é a auditoria ser vista como um processo educativo ${ }^{(12,15,16)}$. A auditoria de enfermagem, como processo educativo fornece importantes subsídios para a implantação e gerenciamento de uma assistência de enfermagem mais segura, ágil, criativa, eficaz e eficiente $^{(15)}$. Os serviços de auditoria de enfermagem necessitam realizar um trabalho proativo visando à redução dos desperdícios com medicamentos e materiais, para isso é importante a revisão das rotinas e implantação de programas de treinamento para conscientização da equipe de enfermagem quanto à importância dos recursos financeiros do hospital ${ }^{(12)}$. Seguindo a mesma linha de pensamento, propõem-se ações de educação continuada sobre os registros de enfermagem para a diminuição dos problemas com as anotações de enfermagem ${ }^{(16)}$.

Dessa forma, existe a necessidade premente de intenso investimento em educação continuada e permanente, visando à promoção do conhecimento e à sensibilização da equipe de enfermagem para a importância da comunicação escrita, e para o fato de que os registros são a expressão do cuidado produzido, ou seja, refletem a qualidade da assistência ${ }^{(26)}$, não tendo apenas o dever de garantir o pagamento pelos procedimentos realizados.

\section{CONSIDERAÇÕES FINAIS}

Considerando a auditoria em enfermagem uma problemática de pesquisa importante e emergente no contexto dos serviços de saúde e a partir da análise realizada, apresentam-se as seguintes considerações e sugestões:

- As pesquisas que vêm sendo desenvolvidas acerca de auditoria em enfermagem no Brasil na última década (1998 a 2008) caracterizam-se como estudos de abordagem quantitativa do tipo descritivo-exploratório ou exploratório, de cunho documental e retrospectivo. Os hospitais ou instituições de saúde vinculadas à iniciativa privada são os principais cenários investigados. 
- Os resultados dos estudos descrevem as principais distorções e não conformidades presentes nas anotações de enfermagem, relacionando-as, principalmente, com a qualidade da assistência de enfermagem e os prejuízos financeiros à instituição. Também destacam a função administrativa do enfermeiro, sobretudo a sua função de liderança junto à equipe de enfermagem e a auditoria de enfermagem como um processo educativo.

- A temática auditoria de enfermagem ainda é pouco explorada na literatura científica nacional. São necessários novos estudos que busquem além de quantificar os erros presentes nas anotações de enfermagem e os prejuízos decorrentes de tais inconformidades, conhecer, discutir e analisar as práticas desenvolvidas pelos enfermeiros com relação à auditoria e os aspectos contextuais que têm interferindo na qualidade dos registros de enfermagem.
- No campo formativo, atividades de educação permanente representam uma boa estratégia a ser empregada, principalmente se conduzirem os trabalhadores de enfermagem a uma reflexão sobre sua prática e as possibilidades presentes no cotidiano do trabalho para realização de uma assistência mais qualificada e pautada na ética, contribuindo, desse modo, para valorização da enfermagem como profissão do cuidado. Além disso, é necessária maior inserção da temática nos projetos pedagógicos dos cursos de formação, tanto ao nível técnico como superior.

- No campo da prática/assistência de enfermagem, é necessária uma reflexão crítica da função-papel do enfermeiro, em especial no âmbito hospitalar, acerca da auditoria não somente como uma ferramenta voltada aos interesses financeiros e políticos das instituições, mas, também como uma estratégia na busca e conquista da qualidade do atendimento e assistência de enfermagem.

\section{REFERÊNCIAS}

1. Pereira LL, Takahashi RT. Auditoria em enfermagem. In.: Kurcgant $\mathrm{P}$, organizadora. Administração em Enfermagem. São Paulo: EPU, 1991. p. 215 -22.

2. Riolino NA, Kliukas GBV. Relato de Experiência de Enfermagem no campo de Auditoria de Prontuário - uma ação inovadora. Nursing (São Paulo) 2003;65(6):35-38.

3. Motta ALC, Leão E, Zagatto JR. Auditoria Médica no Sistema Privado: abordagem prática para organizações de saúde. São Paulo: látria; 2005.

4. Scarparo AF. Auditoria de Enfermagem - identificando sua concepção e métodos. [dissertação]. Ribeirão Preto (SP): Escola de Enfermagem de Ribeirão Preto, Universidade de São Paulo; 2007.

5. Hausmann M. Análise do processo de trabalho gerencial do enfermeiro em um hospital privado no município de São Paulo: possibilidades para o gerenciamento do cuidado. [dissertação]. São Paulo(SP): Escola de Enfermagem, Universidade de São Paulo; 2006.

6. Muñoz WIS, Takayanagui AMM, Santos CB, Sanches-Weatman O. Revisão sistemática da literatura e metanálise: noções básicas sobre seu desenho, interpretação e aplicação na área de saúde. In: Anais do $8^{\circ}$ Simpósio Brasileiro de Comunicação em Enfermagem; 2-3 maio 2002; Ribeirão Preto, Brasil. Ribeirão Preto: Universidade de São Paulo; 2002. [citado em 19 nov 2008] Disponível em: URL: <http://www.proceedings.scielo.br/pdf/sibracen/n8v2/v2a074.pdf $>$.

7. Lopes ALM, Fracolli LA. Revisão sistemática de literatura e metassíntese qualitativa: considerações sobre sua aplicação na pesquisa em enfermagem. Texto \& Contexto Enferm. 2008;17(4):771-8.

8. Bardin L. Análise de conteúdo. Lisboa: Edições 70; 1977.

9. Lopes CM. Auditorias distorções: ênfase nas atividades de anotações de enfermagem. Rev. Bras. Enferm 1998;51(3):105-22.
10. Ochoa-vigo K, Pace AE, Rossi LA, Hayashida M. Avaliação da qualidade das anotações de enfermagem embasadas no processo de enfermagem. Rev. Esc. Enferm. USP 2001;35(4):390-8.

11. Souza V, Moura FL, Flores ML. Fatores determinantes e consequências de falhas registradas na assistência de enfermagem: um processo educativo. REME: Rev. Min. Enf. 2002;6(1/2):30-4.

12. Galvão CR. Estudo do papel da auditoria de enfermagem para a redução dos desperdícios em materiais e medicamentos. Mundo saúde (1995) 2002;26(2):275-82.

13. Rodrigues VA, Perroca MG, Jericó MC. Glosas hospitalares: importância das anotações de enfermagem. Arq. Ciênc. Saúde. 2004; 11(4):210-4.

14. Fonseca AS, Yamanaka NMA, Barison THAS, Luz SF. Auditoria e o uso de indicadores assistenciais: uma relação mais que necessária para a gestão assistencial na atividade hospitalar. Mundo saúde (1995) 2005;29(2):161-69.

15. Souza DA, Fonseca AS. Auditoria em enfermagem: visão das enfermeiras do município de São Paulo. Nursing (São Paulo) 2005;84(8):234-8.

16. Luz A, Martins AP, Dynewicz AM. Características de anotações de enfermagem encontradas em auditoria. Rev Eletrônica Enferm. [periódico online] 2007 [citado 20 set. 2008];9(2):344-61. Disponível em: http://www.fen.ufg. br/revista/v9/n2/v9n2a05.htm.

17. Scarparo AF, Ferraz CA. Auditoria em Enfermagem: identificando sua concepção e métodos. Ver. Bras. Enferm. 2008;61(3):302-5.

18. Venturini DA, Marcon SS. Anotações de enfermagem em uma unidade cirúrgica de um hospital escola. Ver. Bras. Enferm. 2008;61(5):570-77.

19. Brasil. Conselho Federal de Enfermagem. Resolução $n^{\circ}$ 266/2001. Dispõe sobre as atividades do Enfermeiro Auditor. [citado em 20 dez 2009]. Disponível em: 
http://www.portalcofen.gov.br/2007/downloads/ANEXO2662001.pdf.

20. Gil AC. Como elaborar projetos de pesquisa. $4^{\text {a }}$. ed. São Paulo: Atlas; 2007.

21. Leopardi MT. Alguns aspectos da pesquisa quantitativa. In: Leopardi MT, organizadora. Metodologia da Pesquisa na Saúde. Florianópolis: UFSC/Pós-Graduação em Enfermagem, 2002. p.181-191.

22. Yin RK. Estudo de Caso: Planejamento e Métodos. $3^{\mathrm{a}}$ ed. Porto Alegre: Bookman; 2006.

23. Wright JTC, Giovinazzo RA. Delphi: uma ferramenta de apoio ao planejamento prospectivo. Cad Pesqui Adm. 2000;1(12):54-65

24. Camelo, T, Silva-Jr OC. Tratamento do Tema Auditoria de Enfermagem em Base Eletrônica de Dados. Rev. Meio Amb. Saúde 2006;1(1):7-12.

25. Scarparo AF. Auditoria em Enfermagem: revisão de literatura. Nursing (São Paulo) 2005;80(8):46-50.

26. Vituri D, Matsuda L. Analysis of nursing records as to care quality: a retrospective descriptive exploratory study of quantitative approach. Online Braz J Nurs. [serial online]. 2008 [cited $2008 \mathrm{dez}$ 14]; 7(1). Available from: http://www.uff.br/objnursing/index.php/nursing/article/ view/j.1676-4285.2008.1241/295.

27. Campos JF, Souza SROS, Saurusaitis, AD. Auditoria de prontuário: avaliação dos registros de aspiração traqueal em terapia intensiva. Rev Eletrônica Enferm. [periódico online] 2008 [citado $18 \mathrm{dez}$ 2008];10(2):358-66. Disponível em: www.fen.ufg.br/revista/v10/n2/pdf/v10n2a07.pdf. 